\title{
Reflections on a Multi-stakeholder National Campaign in India around Comet ISON
}

\author{
Prajval Shastri ${ }^{1}$ (on behalf of the Eyes on ISON campaign team) \\ ${ }^{1}$ Indian Institute of Astrophysics, Bengaluru 560034 India \\ email: pshastri@iiap.res.in
}

\begin{abstract}
A country-wide awareness campaign was conducted in India on the trail of Comet ISON, which built upon the networks created during IYA2009, thus maximising its reach.
\end{abstract}

A striking trend in modern-day India is the salience of technology at all socio-economic levels. There has not been a concomitant rise in scientific temper, however, and superstitions are prevalent even among the educated or privileged classes. A parallel trend in most schools is that learning by doing and discovering is on the back burner, with an emphasis on rote learning instead. This is despite government policy on education embodied in the National Curriculum Framework 2005 that aims at equality of outcome regardless of socio-economic background, which perforce requires activity-based learning. In this context, a national awareness campaign around Comet ISON was designed, particularly aimed at teachers and students. The campaign built heavily upon the networks and collaborations created preparatory to IYA2009 spearheaded by the Indian Institute of Astrophysics (IIA Newsletters 2009, 2010). The hallmarks of the campaign were:

- It was co-ordinated by a loose consortium of practitioners from professional astrophysics and science communication, amateur astronomy and peoples' science movements,

- It was built around a cascading series of training workshops with a common opensource curriculum, a workshop primer and resource material generated by the campaign,

- The resource material (activity books, posters, multi-media presentations) was generated in several regional languages on the web (and continues to be a long-term resource),

- It leveraged the world-wide web to make the open-source multi-lingual high-resolution material digitised and downloadable, for poster printing and multi-media use,

- Its workshop primer emphaisized inclusive organization and participation.

Details of the team, training workshops, curricula and resource materials are on the Eyes on ISON campaign website (http://ison.metastudio.org) $\dagger$; also $c f$. on-line presentation.

Outputs $\&$ Outcomes. The curriculum for the training workshops included talks on the solar system, comets and the history of Indian astronomy and astrology (along with multi-media presentations); activities on day-time astronomy (along with videos), comet craft, astronomy role playing and night-sky watching (along with activity books); discussion activities on frequently asked questions by the public on astronomy and astrology. The three national-level workshops trained a total of 200 trainers. The 38 state-level workshops conducted in regional languages inturn trained over 2000 train-

$\dagger$ HBCSE-TIFR hosted the brainstorming meeting that kicked off the campaign. Vigyan Prasar, Dept. of Science \& Technology, Govt. of India, funded this meeting and the national-level workshops. The state-level workshops were largely funded by DST-NCSTC. Funds for several state-level and all district-level workshops as well as other events were locally generated. 
ers, who also constitute a network which is a resource for future campaigns. While the events of the campaign were heavily under-reported, at least 130 district-level workshops trained over 5000 communicators country-wide, which includes a large fraction of school teachers. 